\title{
Comparison of a New Automated Kinetically Determined Fibrinogen Assay
}

\begin{tabular}{|l|l|l|}
\hline W.-M. & Walter-Michael & Halbmayer $^{\mathrm{a}, \mathrm{b}}$ \\
\hline M. & Michael & Fischer $^{\mathrm{a}}$ \\
\hline E. & Elisabeth & Legenstein $^{\mathrm{b}}$ \\
\hline E. & Erich & Kaiser $^{\mathrm{b}}$ \\
\hline
\end{tabular}

${ }^{a}$ Central Laboratory, Municipal Hospital Vienna-Lainz, and ${ }^{\mathrm{b}}$ Austrian Society for Quality Assurance and Standardization of Medical and Chemical Laboratory Investigations (ÖQUASTA), Vienna, Austria

Prof. Dr. med. M. Fischer, Zentrallaboratorium, Krankenhaus der Stadt Wien-Lainz, 13, Wolkersbergenstrasse 1, A-1130 Wien (Austria)

Table 1. Range of coefficients of variation (CV) of three different fibrinogen assays (Clauss, kinetic and derived) of the external quality exercises of the years 1994, 1995 and 1996 (February-May) by ÖQUASTA in

Austria

CV Clauss,

Exercises

$(\mathrm{n}=4)$

$(\mathrm{n}=4)$

$(\mathrm{n}=2)$

3.5-13.3 5.8-10.3 3.1-16.0

$\mathrm{n}=$ Number of hemostaseologic exercises per year; data $\mathrm{n}=$ total number of compiled fibrinogen results of exercises per year.

Dear Sir,

We completely agree with Pál László, who mentioned in his letter to the editor 'Comparison of Fibrinogen Determinations' [1] that the most reliable procedure to compare different methods depends on the use of only one (pooled or commercially lyophilized) reference plasma for calibrations. However, and unfortunately, in clinical practice a great variety of heterogenous commercial calibrator plasmas are used [2]. In our paper [3], we used four different and for the different methods recommended calibration materials not in spite of the well-known fact of deviations from the declared value but because of this effect! As mentioned in our paper [3], we did not evaluate or compare four methods but studied the new kinetic method (using analyzer, reference and control plasmas according to the recommendation of the reagent manufacturer) in comparison with those derived from the three most frequently used and best established automated meth-

\section{Data}

\section{4}

ods in Austria (according to the ÖQUASTA, the Austrian Society for Quality Assurance and Standardization of Medical and Chemical Laboratory Investigations). Maybe we should have used the term 'utilizations' instead of'methods' for a better understanding. Since in daily routine the clinical laboratories use the reference plasma recommended (and produced) by the manufacturer of the reagent/

analyzer or the very reference plasma which has been used in the laboratory ever since, the use of one reference material for all 'methods' (e.g. adaptations of fibrinogen determinations) would not reflect the true heterogeneity of fibrinogen determinations in the clinical routine.

CV kinetic, \% CV derived, \%

8.6-10.8

5.4-7.8 6.6-10.5

$5.7-8.6$

$6.2-10.6$

8.47-8.48

This was the reason why we decided to use the complete 'methods' (e.g. adaptations) - in Austria

have been rather similar within the last 3 years (table 1) and denote equally distributed precision of determination among the groups. In contrast, the data of the latest exercises (February and May 1996)

most frequently used - of the fï-brinogen determinations in order to demonstrate that a critical consideration of fibrinogen values by the clinical physician with dependence on an acute-phase reaction and the type of fibrinogen assay (e.g. utilization or adaptation) is required as well as it is desirable to specify the method with each fibrinogen result in the laboratory report. Meanwhile several manufacturers are trying hard to produce a reliable fibrinogen standard plasma preparation which is applicable to all methods. 
It might be of interest that the data of the external quality assurance exercises by the ÖQUASTA in Austria confirm the finding of elevated fibrinogen levels using the derived method although this effect might be due to the fact that for ÖQUASTA exercises lyophilized plasma samples are used:

Each year, the ÖQUASTA organizes and coordinates 4 hemo-staseologic exercises in which the data of approximately 150 Austrian laboratories interested in hemosta-sis are compiled and evaluated [4]. The coefficients of variation among the fibrinogen methods 'Clauss', 'kinetic' and 'derived'

Table 2. Results (number of participants, mean \pm 1 SD) of the two ÖQUASTA exercises in 1996 (February and May) of the three most frequently used fibrinogen assays in Austria

Clauss

Kinetic

Derived

$\mathrm{n}$ mean \pm 1 SD $n$ mean \pm 1 SD $n$ mean \pm 1 SD

Exercise February 96

Reagent 1 Reagent 2 Reagent 3 Reagent 4 Reagent 5

$56 \quad 247+18$

$22 \quad 234 \pm 7$

$30 \quad 255 \pm 22$

$5 \quad 242 \pm 11$

$20 \quad 249 \pm 17$

Exercise May 96

$17245+21 \quad 15357 \pm 23$

$54 \quad 307 \pm 32$

$26 \quad 279+18$

$26 \quad 307 \pm 22$

$5 \quad 301 \pm 45$

$21 \quad 300 \pm 33$

Reagent 1 Reagent 2 Reagent 3 Reagent 4 Reagent 5

$18308 \pm 26 \quad 15370 \pm 39$

$\mathrm{n}=$ Number of returned data (results) by participants of the exercise.

demonstrate higher mean levels of the derived fibrinogen method in 15 laboratories when compared to the Clauss or the kinetic method even after splitting the data according to the used reagent (table 2).

References

3 Halbmayer W-M, Haushofer A, 4 Halbmayer W-M, Legenstein E,

László P: Comparison of fibrinogen determinations. Haemostasis 1996; 26:178.

Furlan M, Felix R, Escher N, Lämmle B: How high is the true fibrinogen content of fibrinogen standards? Thromb Res 1989;56: 583-592.

Schön R, Radek J, Fischer M: Comparison of a new automated kineti-cally determined fibrinogen assay with the 3 most used fibrinogen assays (functional, derived and nephe-lometric) in Austrian laboratories in several clinical populations and healthy controls. Haemostasis 1995; 25:114-123.

Kaiser E: External quality assurance: An essential element of the quality management in clinical laboratories. Wiener Klin Wochenschr 1995;107:500-506.

50

Haemostasis 1997;27:49-50

Halbmayer/Fischer/Legenstein/Kaiser 